\title{
What happens to computer science research after it is published? Tracking CS research lines
}

Jacques Wainer, Eduardo Valle

August 14, 2012

\begin{abstract}
Are computer science (CS) papers extended after they are published? We have surveyed two hundred computer science publications, one hundred journal articles and one hundred conference papers, using self-citations to identify potential and actual continuations. We are interested in determining the proportion of papers that do indeed continue, how and when the continuation takes place, and whether any distinctions are found between the journal and conference populations. Despite the implicit assumption of a research line behind each paper, manifest in the ubiquitous "future research" notes that close many of them, we find that more than $70 \%$ of the papers are never continued.
\end{abstract}

\section{Introduction}

A sketch of future research directions has become a customary closing for research papers in computer science. For example, in a set of 100 conference and 100 journal papers (discussed below), 41 of the conference papers and 37 of the journal papers had either a "future research" section, or statements in the form "we plan to extend this research by (...)". That practice reveals an implicit, but pervasive assumption of scientific production in the field of computer science (CS): that behind each article there is a research line, which persists after publication is accomplished. However, many authors never fulfill that hint at continuation, and many papers are the terminuses of their research lines. How many do effectively continue? Does it make a difference whether the paper was published in a journal or in a conference? Does the chance of continuation correlate with the paper reception by the community?

Those are some of the questions we are interested in answering. We sampled independently journal and conference papers, and used self-citations to those papers to explore how they were extended by their authors. The underlying assumption is that, in a research line, follow-up papers will cite the previously published ones, making self-citations useful to track the continuations.

By contrasting the journal and the conference samples, we also intend to contribute to the ongoing debate about the roles of those media on the field of computer science. Is research more likely to continue when it is published in conferences, and thus do conferences play the same role in CS as they do in other disciplines, as places to report ongoing efforts and receive feedback from the community? Or is publication in conferences actually less likely to continue, suggesting that CS conferences are places to publish less elaborated, more speculative (and thus riskier) work?

We are interested in not only quantifying how much of the work in CS continues after publication, but also in how does that continuation proceed. Specifically, we want to know:

- how many research lines continue after that publication?

- how do the continuations split between journals and conferences?

- how long does it take for the next paper in a research line to be published?

- who pursues the continuation - roughly the same group, or just one author? Are the senior or the junior researchers most often leading the continuations?

- is there a correlation between the impact (citation count) of the original paper and the fact the research 
line continues?

- is there a correlation between the quality or selectivity of the original medium and the fact the research line continues?

For each of those questions, we are interested in the differences that might exists between originals published in conferences and in journals; and what those differences tell us about those two media.

\section{Related research}

\subsection{Conferences and Journals}

In the discipline of computer science, the role of conferences and journals as publication media has been a contentious subject (Fortnow, 2009; Freyne, Coyle, Smyth \& Cunningham, 2010, Halpern \& Parkes, 2011; Wing and Guzdial, 2009).

In most scientific fields, conferences aim at promulgating works in their earlier development stages. Therefore, the review requirements are lower, and the proceedings, when they are produced at all, are not intended for archival and reference use. That is conspicuous in the much lower citation count conference proceedings receive, when compared to journal articles: for the aggregation of all disciplines proceedings attract less than $5 \%$ of all citations, a fraction that has been steadily decreasing (Lisée, Larivière \& Archambaud, 2008).

However, in engineering, and especially in computer science, the situation is not obvious. The traditional evolutionary model from early drafts as conference proceedings to mature work as journal articles is no longer clearly observable. In stark contrast to other disciplines, proceedings get a large — and growing fraction of all citations (Lisée et al., 2008). That contrast is further marked by the fact CS conferences have a more stringent review process than most disciplines, sometimes being extremely selective (Fortnow, 2009).

The reasons for that relatively privileged status of conferences in computer science are not completely understood. Some authors have pointed to the accrued importance of timeliness in a rapid evolving field such as computer science, making conferences preferable (Montesi \& Lago, 2008). However, the emphasis of the field on conferences has come under growing criticism, on the grounds that it leads to hasty peerreview, deadline-driven research, and deprivation from conferences of their usual role of fora for debate and exchange of ideas (Grudin, 2011; Wing \& Guzdial, 2009).

Lisée et al. (2008) collect citations spanning the years 1980-2005 from the Thomson Scientific Science Citation Index (SCI), Social Sciences Citation Index (SSCI) and Arts and Humanities Citation Index (AHCI). They analyze, thus, references made by "archival" material (articles, notes and review articles), isolating among those references to conference proceedings. Only the references are analyzed (checking for terminology typical of proceedings), the source documents are not verified. In case of doubts, the reference is rejected as noise. They find that from 1980 to 2005, the fraction of references to conferences steadily decreases from about $2.3 \%$ to about $1.7 \%$ of all citations. This is in stark contrast of the disciplines of engineering and computer science, where that fraction has grown from $7.6 \%$ to $9.4 \%$ in the same period. In the subfields of natural sciences and engineering, they identify computer science as the one with the largest fraction of references to conferences (about 20\%), followed by electrical engineering and electronics (with about $13 \%)$.

Wainer, Oliveira, and Anido (2011) analyze the references made by computer science conference papers (in 2006). $40 \%$ of the references are to conference papers, and $30 \%$ to journal papers. Furthermore of all documents cited at least 10 times by all conference papers published by the Association for Computing Machinery (ACM) in 2006, 41\% are also conference papers, while only 13\% of them are journal papers.

Montesi and Lago (2008) work on the narrower discipline of software engineering, examining how 
conference papers are extended for publication in journals. They survey both a sample of 22 editors of major publications in software engineering, using semi-structured interviews, and a sample of 122 authors, using a Web questionnaire. They find that editors expect authors to submit extended versions to journals, and show disappointed that authors are "too often content with their conference submission". On the other hand, they expect significant extensions, careful updating of the references list, and willingness to completely rewrite the article — instead of just adding a few sections — in order to address the needs of a different audience.

There is an ongoing debate on the role of conferences in journals in CS scholarly publications. The subject has appeared frequently in the pages of ACM Communications, one of the main showcases of the discipline, starting with an editorial by its editor (Vardi, 2009).

In contrast to what happens in many disciplines, CS conferences are refereed, and some of them are extremely selective. Such selectivity, according to Fortnow (2009), is not without drawbacks, since conferences cannot afford the careful and well-paced review process of journals. The results, according to him, is an emphasis on safe (incremental and technical) papers, the introduction of random factors in the

judgment (because of limited discussion between the authors and the program committee), and a disposition to deadline-driven research, cut into "least publishable units".

Halpern and Parkes (2011) further discuss the ideal role of conferences and journals in CS publication, proposing that conferences should be used for rapid dissemination, and journals for certification, instead of the current model, where conferences hold both roles. They discuss what is needed in order for that change of attitude to take place, remarking, for example that CS journals need to become much faster in reviewing papers if they are to play a more prominent role.

It is clear that the classical evolutionary model fails to account for the publication conventions of computer science. What is not clear is exactly in which aspects the current practice deviates from that model, and why. We believe the current debate is hindered by the fact the process leading to journal articles from conference proceedings in Computing disciplines has been largely unexplored (Montesi \& Lago, 2008).

Our goal here is to explore that process. Of course, explaining how and why computer science has attained their atypical publishing practices is a complex task, which we have no pretense to exhaust. We aim instead at keeping a neutral point of view, and focusing on data gathering and statistical analysis. We expect that our data will help to advance the current discussions, providing a better understanding of the status quo, revealing unsuspected patterns, and grounding the intuitive ones in hard data.

\subsection{Self-citation Practices}

Previous research has not placed the emphasis, as we do, in self-citations as indicative of potential continuations of research lines, but in quantifying the fraction of all citations they represent. Mainly, the motivation has been to evaluate their impact in scientometric and bibliometric measures, including individual authors' impact measures.

Aksnes (2003) studied self-citations in scientific papers published by authors in Norway. They studied 46,849 papers published from 1981 to 1996, and verified the citations to those papers up to the year 2000 . On the average $21 \%$ of the citations made to those papers were author self-citations. They studied different scientific fields, and for computer science, the topic of this paper, they discovered that $24 \%$ of the citations made to the CS papers in the set was self-citations. Hyland (2003) also analyses self-citations, or more particularly self-references (citations made by the paper to previous work by the same authors) using a set of 240 articles published in leading journals in 8 disciplines. That set did not include computer science, but for the two closest disciplines represented, mechanical and electrical engineering, Hyland (2003) finds that that $11.8 \%$ and $9.3 \%$ of the references in the papers respectively were to works by the same authors.

Other authors (Fowler \& Aksnes, 2007; van Raan, 2008) are more interested in the impact of self-citations in bibliometric measures, such as the h-index and the total citation count. Finally, some authors (Frandsen, 2007) are concerned with the practice of self-citation at the journal level, i.e. the practice (maybe 
encouraged by publishers) of papers in a journal citing other papers in the same journal, to inflate its impact factor.

Although the comparison of publishing practices (including citation practices) among disciplines has been a topic of interest (Lisée et al., 2008; Montesi \& Lago, 2008), the continuation of research lines is a novel angle to those investigations. We are unaware of previous studies in any scientific discipline, except for the work of Eckmann, Rocha and Wainer (2012), which also relates to computer science. That study bears some similarities to this one, but their focus is the relationship between papers accepted in top-rated computer vision conferences, which were later published in top-ranked journals. The aim was to understand how much the publication in a top conference was a requirement to publishing in the top-rated journals, both as indicated by data, and as explicitly expressed by community opinion. Although the emphasis was not on the continuation of a research line, clearly the conference published papers were either extended or continued in the later journal publications. The study proposed here aims both at a broader scope (computer science as a whole, instead of just computer vision) and more general questions.

Duplicate publication is a tangential issue to our work: our aim is not to analyze republications, but we were aware of the phenomenon, since some of the self-citations turned out to fall in that category, especially on the conference originals sample. Therefore, for the sake of completeness, we cursorily mention that studies on duplicate publication can be found mainly for medical sciences (von Elm, Poglia, Walder \& Tramer, 2004), but there is one study focusing on technological areas (Kostoff et al., 2006). Duplicate publishing generates an array of issues, like the inflation of some of the indices used for merit evaluation, not to mention the risk of copyright disputes, because often some of the authors are omitted in the republication, or the copyright of the original work belongs to the publisher. In addition to that, the medical literature is particularly interested on how duplicate publication of the same experimental data might bias systematic reviews and meta-analyses of a particular topic.

\section{Data and Methods}

The Association for Computing Machinery (ACM) is the world's largest educational and scientific computing society, having over 100,000 members, sponsoring over 170 conferences and publishing more than 50 journals, magazines and newsletters ${ }^{1}$. We have selected a random sample of 100 papers published in ACM sponsored conferences and 100 papers in ACM sponsored journals, all in the year of 2003. The data collection took place in October 2008 for the conference papers, and in November 2011 for the journal papers, both from the ACM Digital Library (ACM DL). The aim in both samples was selecting full, peerreviewed, scientific articles, representative of a large array of CS disciplines. Therefore, articles with 4 pages and less were removed, to avoid considering extended abstracts, short papers (published in conferences) and editorials (published in journals). For the journals sample, we have removed both the ACM newsletters (e.g., ACM SIGPLAN Notices) and the magazines (e.g., Communications or Interactions), which have many regular columns and invited articles. For the journal sample, we have excluded two atypical cases: the very large volume 22 issue 3 from Transaction on Graphics (TOG) which published the 2003 SIGGRAPH proceedings, and the Journal of the ACM (JACM) volume 50 issue 1, which published a commemorative issue for its 50 years, with many invited articles. We have called the set of 100 conference papers the conference originals and the set of 100 journal papers the journal originals. Collectively they were called originals.

We collected the citations to the originals, also using the ACM Digital Library (DL), in November of 2011. The papers that cite the originals, and that were co-authored by any of the original's authors were considered follow-ups. All papers were taken into account for the follow-up analysis, including short ones — provided they were more than a simple abstract. Note also that the follow-ups come from "The Guide to Computing Literature" of ACM DL, which includes journals and conferences from many publishers, comprising thus citations from CS at large. We have then analyzed each follow-up to verify if indeed they 
were true continuations of the originals. After examining a few dozen samples, we devised a three-way classification of the self-citations for the follow-ups:

- state of the art: the original paper was listed as part of the previous art in the follow-up paper, but was not otherwise related to it. In those cases, the original work was cited among others in the related research section of the follow-up, with or without any further elaboration. For example the citation to "Eckmann, Rocha and Wainer (2012)" in the previous section is a state-of-the-art self-citation. That paper was used in the related literature section to situate this research in the context of previous work, but this research does not continue that one, although they are both in the general area of conference and journal publications in CS.

- extension: if the follow-up paper uses or extends the original paper.

- republication: if the follow-up is the same paper as the original, allowing for formatting and minor text differences. It also includes papers with deletions from the original, but no new material.

We have analyzed the papers by looking mainly at the context of the citations to the originals and the papers' abstracts, but we have often looked elsewhere in the full text, when those evidences were not sufficient to make a decision. Each of us, the authors, has analyzed the papers independently, and where we have disagreed on the classification, we have discussed our points of view, until a consensus was reached. The disagreements happened in a minority of cases, and normally we could reach consensus quickly, pointing out at some evidence the other author had overlooked. Just a handful of borderline cases have taken, each, a few dozen minutes to conciliate.

Our classification tries to represent a spectrum of relatedness that goes from weakly related (state of the art) to almost exact reproduction (republication). However, only when the self-citation falls in the extension class, we have considered that a follow-up was a true continuation, calling it an extension as well.

For all extensions of the originals, we have also collected data regarding the time of publication, the number of authors in common with the original, and whether the continuing authors were junior or senior researchers. The status of an author as junior or senior was determined by looking at the author data in the ACM DL. If the author had any paper listed with publication date on or before 1998 (5 years before the publication of the originals) he or she was considered a senior researcher, otherwise, a junior one. Since a $\mathrm{PhD}$ usually lasts around 5 years, and PhD students are the ones we would like to consider junior researchers, that interval was used to distinguish them from their advisors, considering unlikely that a typical $\mathrm{PhD}$ student would have publications before their graduate study years. That collection of junior/senior data was performed in November 2011.

We have also collected data on the "quality" or selectiveness of the original conferences and journals. The quality of the conference was determined by verifying the publication data about the conference in the ACM DL. From the list of all ACM proceedings ${ }^{2}$, links to specific conferences provide, in most cases, the total number of citations, the total number of papers accepted, and the acceptance rate. When there were no data for the acceptance rate on the year 2003, we have used the data for the earliest date available after 2003. For 8 of the conferences represented in the sample, no data were available at all, so we have removed those conference from the calculations related to "quality." The metrics of quality employed are both the rejection rate and the average number of citations received by the papers published in the conference that year. Rejection rate is an a priori measure of quality of a conference. One expects that conferences with higher rejection rates are selecting better papers to be published, although Freyne et al. (2010) have recently shown that for the rejection rates and the impact factors of particular conference venues (each year being a data point) correlate very poorly. Citations per paper is an a posteriori measure. If the conference had indeed "better" papers, they should receive, on average, more citations.

The "quality" of the journals was determined by using Thompson Reuters' journal impact factors, as 
published in the 2003 Journal Citation Records report. Unfortunately, of the 19 different ACM journal represented in our sample, 8 had no Thompson Reuters' impact factors in 2003. As we did for conferences, we removed those 8 journals from the calculations.

Finally, the acknowledgment of the research by the CS community was measured by whether the paper had received at least one citation that was not a self-citation, i.e., from a paper that did not include any of the original co-authors of the paper. Although not perfect, we have used that as a metric that the paper was "accepted" outside its original group.

\subsection{Statistical Analysis}

Our goal is to estimate certain parameters of the entire publication population from our sample. The standard way is to report confidence intervals for those estimations, reflecting interval of values one expects (with a certain confidence) the population parameter will fall, given the measures taken in the sample.

Employing the confidence of $90 \%$, we compute the confidence intervals for proportions, using the pseudoconfidence interval for multinomial distributions, implemented by the function gofCI in the package NCStats for the statistical software R. Confidence interval for means are computed using the standard ttest ( $t$. test in $R$ ), and for the confidence intervals of medians we use a bootstrapping procedure with 10,000 repetitions (boot. $\mathrm{C} i$ in $\mathrm{R}$ ). We abbreviate the $90 \%$ confidence interval as $(\mathrm{CI}=\mathrm{a}, \mathrm{b}$ ), where a and $\mathrm{b}$ are, respectively, the lower and upper limit of the interval. For the correlation studies we employ a linear regression, considering models and parameters significant when their p-value is below 0.10 (reflecting, again, $90 \%$ confidence).

\section{Results}

We start by presenting the results of our survey, without any commentary beyond the statistical analysis. We invite the reader to think about these data and their meaning, before looking at our own interpretations and conclusions in the next section.

There were 7134 proceedings articles published by ACM in 2003, which roughly correspond to our population of conference originals. We say roughly because the original set contained papers with less than 4 pages, which were excluded from our sample. From the surviving ones, we have sampled 100. There were 533 journal papers published in 2003, which again corresponds roughly to the population, except that this set contains the articles published in the excluded TOG and JACM issues, besides the papers with less than 4 pages, which were excluded, as before. Again, from the surviving ones, we have sampled 100. There were 20 different journals published in 2003, from which 19 appeared in the sample; and there were 147 different conferences, from which 44 turned out in the sample.

Table 1: How many follow-ups are expected? For each sample (conferences and journals) we tabulate how many originals have produced a certain number of follow-ups.

\begin{tabular}{c|c|c|c|c}
\hline \multirow{2}{*}{ \# of follow-ups } & \multicolumn{2}{|c|}{ Conferences } & \multicolumn{2}{c}{ Journals } \\
\cline { 2 - 5 } & \# of originals & $90 \%$ CI* & \# of originals & $90 \%$ CI* \\
\hline 0 & 44 & $36-52$ & 51 & $43-59$ \\
1 & 28 & $21-36$ & 49 & $41-57$ \\
2 & 11 & $7-17$ & & \\
3 & 7 & $4-12$ & & \\
4 & 5 & $2-10$ & & \\
5 & 3 & $1-7$ & & \\
6 & 1 & $0-4$ & & \\
$>6$ & 1 & $0-4$ & & \\
\hline
\end{tabular}

$* 90 \%$ confidence interval on the number of originals. 


\subsection{Follow-ups}

A large fraction of papers - about half of them — does not even have a follow-up. From the 100 conferences papers sample, 44 have no follow-ups. That fraction is even larger for journal papers: 51 from the 100 sampled. A small number of conference papers had several follow-ups, up to 14 for one of the originals. That did not happen for the journal papers: the follow-ups, if present at all, were at most one per original. The entire distributions for both samples can be seen at Table 1.

For the 100 conference originals, the 127 follow-ups were divided evenly between journal (63) and conference (64) publications. For the journal originals, these 49 follow-ups were divided into 28 journal papers, and 21 conference papers ( 3 of which appeared in ACM newsletters that published conference proceedings, being thus considered conference papers).

Table 2: How many extensions are expected? For each sample (conferences and journals) we tabulate how many originals have produced a certain number of true continuations.

\begin{tabular}{c|c|c|c|c}
\hline \multirow{2}{*}{ \# of extensions } & \multicolumn{2}{|c|}{ Conferences } & \multicolumn{2}{c}{ Journals } \\
\cline { 2 - 5 } & \# of originals & $90 \%$ CI* & \# of originals & $90 \%$ CI* \\
\hline 0 & 70 & $62-77$ & 77 & $71-85$ \\
1 & 16 & $11-23$ & 21 & $15-29$ \\
2 & 9 & $5-15$ & & \\
3 & 3 & $1-7$ & & \\
4 & 1 & $0-4$ & & \\
5 & 1 & $0-4$ & & \\
\hline
\end{tabular}

$* 90 \%$ confidence interval on the number of originals.

\subsection{Extensions}

The analysis of the 127 follow-ups for the conference originals, and the 49 follow-ups for the journal originals, revealed, as expected, that not all self-citations are examples of research continuation. In particular, there were 57 cases of state-of-the-art and 15 cases of republication citations among the followups of conference originals ( 28 and 0 , respectively, for the follow-ups of journal originals). For two of the follow-ups of journals, it was impossible to access the full text in order to evaluate them.

Table 2 lists the extension counts for the journal and conference originals. The 52 extensions of the conference originals were divided into 23 that were published in journals, and 29 in conferences. The 21 extensions for the journal originals were divided into 14 journal papers, and 7 conference papers (including 3 published in newsletters as discussed above).

Time to first extension

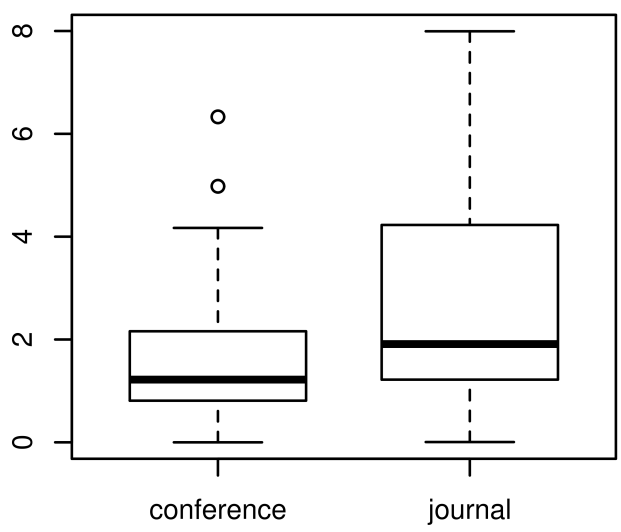


Figure 1: The boxplot shows the distribution, for each sample, of the time elapsed (in years) between the publication of the original and the publication of the first extension.

\subsection{Lapse between original and extension}

The time interval between the originals and extensions is shown in Figure 1, in the form of a boxplot for each population of originals. The boxplot showcases the medians, quartiles and range of the populations. The whiskers extend to the most extreme values until twice the interquartile range. Values outside that scope are considered outliers and plotted individually as small circles.

In addition, we have computed the averages and confidence intervals. For originals published in conferences the average interval was 1.7 years $(\mathrm{CI}=1.3,2.2)$. Breaking up that average further, it takes 1.4 years $(\mathrm{CI}=0.7,2,2)$ if the extension is published in a journal (9 cases), and 1.8 years $(\mathrm{CI}=1.2,2.4)$ if the extension is published in conference. For journal originals, the average time for the first extension was 3.0 years, $(\mathrm{CI}=2.0,3.9)$, with 3.2 years $(\mathrm{CI}=1.2,5.2)$ when the extension was published in a conference, and 2.8 years $(\mathrm{CI}=1.7,4.0)$ when it was published in a journal.

\subsection{Who continues the work}

For conferences, on average, $62 \%(\mathrm{CI}=55,68)$ of the authors of the original were also authors of the continuation. When we consider only the senior researchers in the originals, $65 \%(\mathrm{CI}=56,73)$ of them continue as authors in the extension. For junior authors, $58 \%(\mathrm{CI}=48,67)$ of them remain as authors in the extension. There were 24 extensions in which only one of the original authors remained, and in these cases, 10 were extended exclusively by the junior author.

For journals, $55 \%(\mathrm{CI}=44,65)$ of the authors remained as co-authors of the extensions. The continuation rates for senior authors was $62 \%(C I=49,74)$ and for juniors $37 \%(C I=19,58)$. Of the 10 cases in which only one author remained, only one of them was extended by the junior author.

\section{Citations received}

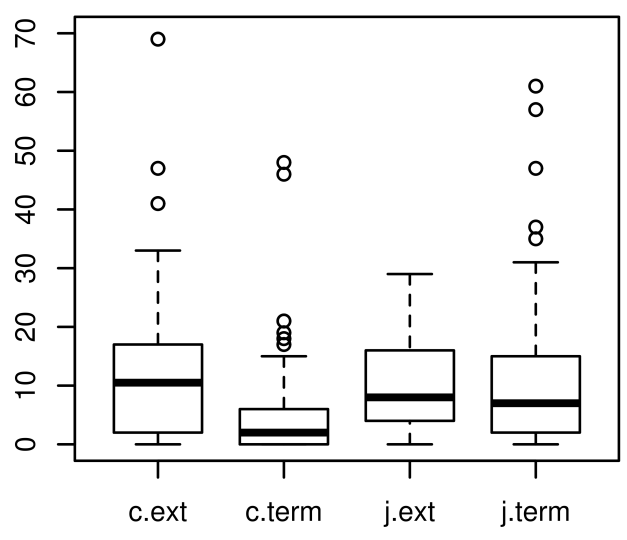

Figure 2: The boxplot shows the distribution of the number of citations (excluding self-citations) received by conference originals with (c.ext) and without (c.term) extensions, and journal originals with (j.ext) and without (j.term) extensions. A few of the most extreme outliers were omitted to avoid compressing the vertical scale too much (188 citations for conference originals with extension; 439, 259, 134, and 130 citations for journal originals without extension; 130 citations for journals with extension).

\subsection{Acknowledgement by community vs. extension by authors}

We contrast four populations of originals in the boxplot of Figure 2: conference and journal originals, subdivided further into those that were extended, and those that were not. For each population, we plot the 
number of citations (excluding self-citations). Outliers twice the interquartile range from the median are indicated by small circles. In addition, some extreme outliers are omitted from the figure, to avoid compressing too much the vertical scale.

The median citation count for conference papers with extensions is $10.5(\mathrm{CI}=6,13.5)$, while for papers without extension, the median is $2(\mathrm{CI}=1,2)$. Thus, for the conference population, the difference appears significant, both visually in the plot and statistically.

For journal originals, the median citation count for papers with extensions is $8(\mathrm{CI}=4,13)$, and for papers without extension, the median is $7(\mathrm{CI}=4,7)$. Both the plot and the statistical analysis indicate that there is not much difference.

For the conference originals, 3 papers out of the 30 with extensions had no citations other than selfcitations, something that happened for 20 out of the 70 papers without extensions. For the journal originals, the numbers are 1 out of 21 for the papers with extensions, and 8 out of 77 for the others.

\subsection{Quality of the medium}

We have investigated the correlation between the proportion of papers that were extended against some measure of quality of the conference or journal. For the conferences we have employed the acceptance rate and the citation count per paper (for all papers in the conference, not just the ones in our sample) as quality metrics. For the journals, we have used the impact factor.

In none of the cases, the regression analysis could find a significant correlation between the quality metric and the proportion of extensions (the p-values found were typically below 0.60 ).

\section{Conclusion and discussion}

We can now address the questions put forth in the introduction of this paper.

Part of the answers comes straightforwardly from the data, like how many papers do effectively continue. We find out that only $23 \%$ (journal) and $30 \%$ (conferences) of the papers published in CS have extensions, indicating a continued line of research. Those numbers are surprisingly low, but as we have mentioned, we lack similar results on other scientific disciplines to properly evaluate those proportions. The presence of a "future works" section or other textual indicatives of continuation seems to be a poor predictor of actual continuation: from the 41 conference papers with such suggestions in the text, only 11 are effectively extended; the proportion for journal papers is of 7 extensions for 37 papers with textual hints of future work.

The difference seems to disfavor journal articles, but it is not significant at $90 \%$ confidence level. However, a significant difference is found in the potential number of extensions, since, when they do continue, conference papers tend to generate more continuations than journal papers.

Leaving the realm of hard data for that of interpretation, we conceive two explanations for that difference. One, more traditional, is that conferences are being used for work in earlier stages, thus, prone to more ulterior development. However, since there is no significant difference in the proportion of the extensions, just in their quantity, we tend to believe that a better explanation might be found considering both the contingencies of conference publishing, and the habits of the CS community. The former, due to stringent deadlines and paper length limitations, may stimulate the research being cut into "least publishable units". The latter possibly makes a CS researcher reluctant in publishing a journal paper that does not duly explore some of its obvious extensions. CS researchers usually favor long and complete journal papers, while conference papers are almost always shorter. Thus, a journal paper would already have exhausted some of its extension avenues.

That hypothesis would also explain the longer time it takes for a journal original to have an extension published. As observed, the elapsed time for the publication of the extension is much shorter for conference 
originals, regardless if the extension is in a conference or in a journal.

Conferences tend slightly more to be extend in conferences than in journals (odds of 1.2 to 1), while journals are much more prone to being extended to other journals (odds of 2 to 1 ). That hints at the directionality of the traditional evolutionary model, which has not been completely abolished, even in the CS community.

For conferences and journal papers, $62 \%$ and $55 \%$, respectively, of the original authors remain as coauthors of the extensions. When researchers do drop-off, the ones who remain tend to be the senior ones. That fact, which somewhat surprised us, suggests that the research lines are "owned" by the senior researchers, who develop it in cooperation with other faculty members and students. It is uncommon for the opposite situation to happen, that is, the students to "carry away" the research line with them, after graduation.

Does the chance of continuation correlates with the community reception of the paper? The correlation is significant for conference papers, but not for journal papers. Of course, correlation does not imply causation, but one can argue that researchers in some level gauge the reception to their conference papers on deciding on whether to continue that line of research. A similar correlation was found by Eckmann et al. (2012) - papers published in top computer vision conferences and then, later, in top journals, had more citations than papers from the same conferences that were not extended to journals. It is less clear why that is not observed on journal papers - one can speculate that the publication delay and the time to accumulate citations are longer for journals, added to the fact that one seldom gets feedback from peers for journal papers. Thus the validation, or lack thereof, provided by journals may not be timely enough to guide the researchers in their decision to continue or not the research line.

More surprising is the lack of correlation between the quality or selectivity of both journals and conferences and the amount of papers that continue. The correlation observed for conference papers in terms of individual impact is not observable in terms of the aggregate metric provided by either the conference selectivity or its collective impact factor. We were expecting either a positive correlation (meaning that the acceptance stimulates the authors to further pursue the research), or a negative correlation (meaning that the publication crowns the research line, liberating the authors to other pursuits). Is it possible that both effects happen in balance, creating a net effect of no correlation?

Finally, let us discuss the $70-77 \%$ of papers that have no continuations. Do they reflect an accomplished result, the terminus of a fruitful research line, or a speculative attempt that did not succeed and was abandoned? Again, we enter the realm of interpretation, and the criterion we have elected is not meant to be waterproof. We consider the research line "accomplished" if that not-continued paper has at least one citation by papers outside the original group, i.e., if it was considered relevant enough by outsiders to be cited at least once. That criterion is, of course, arbitrary, and at once too stringent (a paper can be inspiring and end up not being cited) and permissive (a paper can be cited to be criticized or rebuked). Yet, though we feel the "one external citation" criterion is useless to judge the merit of any individual paper, we believe it is useful as a statistical tool, since the extreme cases get smoothed out.

The figures indicate that conferences are indeed, even in computer science, used for more speculative ideas, with $29 \%(\mathrm{CI}=20,39)$ of papers $(20$ out of 70$)$ without citations other than self-citations. That proportion is much smaller for journal papers, $10 \%(\mathrm{CI}=5,18)$ of papers $(8$ out of 77$)$ in that situation. Again, that hints at the presence of the evolutionary model, even if diluted, in CS.

A non-negligible fraction of authors follows the standard practices of other disciplines, by publishing first in a conference and then following-up in periodicals: $26 \%(\mathrm{CI}=19,34)$ of all conference originals were either extended or republished in journals. The converse phenomenon (extending or republishing journal originals on conferences) happened much more rarely. 


\section{Acknowledgements}

J. Wainer thanks the CNPq for the scholarship grant 301204/2010-1. E. Valle thanks FAPESP. Both authors thank ACM for granting access to the ACM Digital Library metadata in machine-readable format.

\section{References}

Aksnes, D. (2003). A macro study of self-citation. Scientometrics, 56(2):235-246.

Eckmann, M., Rocha, A., \& Wainer, J. (2012). Relationship between high-quality journals and conferences in computer vision. Scientometrics, 90(2):617-630.

Fortnow, L. (2009). Time for computer science to grow up. Communications of the ACM, 52(8):33-35.

Fowler, J. \& Aksnes, D. (2007). Does self-citation pay? Scientometrics, 72(3):427-437.

Frandsen, T. (2007). Journal self-citations: Analysing the JIF mechanism. Journal of Informetrics, 1(1):4758.

Freyne, J., Coyle, L., Smyth, B., \& Cunningham, P. (2010). Relative status of journal and conference publications in computer science. Communications of the ACM, 53(11):124-132.

Grudin, J. (2011). Technology, conferences, and community. Communications of the ACM, 54(2):41-43.

Halpern, J. \& Parkes, D. (2011). Journals for certification, conferences for rapid dissemination.

Communications of the ACM, 54(8):36-38.

Hyland, K. (2003). Self-citation and self-reference: Credibility and promotion in academic publication. Journal of the American Society for Information Science and Technology, 54(3):251-259.

Kostoff, R., Johnson, D., Antonio Del Rio, J., Bloomfield, L., Shlesinger, M., Malpohl, G., and Cortes, H. (2006). Duplicate publication and "paper inflation" in the fractals literature. Science and Engineering Ethics, 12(3):543-554.

Lisée, C., Larivière, V., \& Archambault, E. (2008). Conference proceedings as a source of scientific information: A bibliometric analysis. Journal of the American Society for Information Science and Technology, 59(11):1776-1784.

Montesi, M. \& Lago, P. (2008). Software engineering article types: An analysis of the literature. Journal of Systems and Software, 81(10):1694-1714.

van Raan, A. (2008). Self-citation as an impact-reinforcing mechanism in the science system. Journal of the American Society for Information Science and Technology, 59(10):1631-1643.

Vardi, M. (2009). Conferences vs. journals in computing research. Communications of the ACM, 52(5):55.

von Elm, E., Poglia, G., Walder, B., \& Tramer, M. R. (2004). Different patterns of duplicate publication an analysis of articles used in systematic reviews. JAMA, 291(8):974-980.

Wainer, J., Oliveira, H. P., \& Anido, R. (2011). Patterns of bibliographic references in the ACM published papers. Information Processing \& Management, 47(1):135-142.

Wing, J. \& Guzdial, M. (2009). CS woes: deadline-driven research, academic inequality. Communications of the ACM, 52(12):8-9. 
ENDNOTES

http://www.acm.org/about/fact sheet (accessed 08/2012)

2 http://dl.acm.org/proceedings.cfm (accessed 08/2012) 